\title{
Cell response to oxidative stress induced apoptosis in patients with Leber's hereditary optic neuropathy
}

\author{
C Battisti, P Formichi, E Cardaioli, S Bianchi, P Mangiavacchi, S A Tripodi, P Tosi, A Federico
}

J Neurol Neurosurg Psychiatry 2004;75:1731-1736. doi: 10.1136/jnnp.2003.024372

See end of article for authors' affiliations .....................

Correspondence to: Professor A Federico, Department of Neurological and Behavioural Sciences Policlinico "Le Scotte", V. le Bracci, 2, 53100 Siena, Italy; Federico@unisi.it

Received 18 August 2003 In revised form

10 February 2004 Accepted

23 February 2004

\begin{abstract}
Objectives: Leber's hereditary optic neuropathy (LHON) is a maternally inherited disease in which acute or subacute bilateral visual loss occurs preferentially in young men. Over $95 \%$ of LHON cases are associated with one of three mitochondrial DNA (mtDNA) point mutations, but only $50 \%$ of men and $10 \%$ of women who harbour a pathogenetic m+DNA mutation develop optic neuropathy. This incomplete penetrance and preference for men suggests that additional genetic (nuclear or mitochondrial) and/or environmental factors must modulate phenotype expression in LHON. A role for reactive oxygen species (ROS) in mitochondrial diseases, secondary to mtDNA mutations, or as a result of the direct effect of ROS cytotoxicity, has been implicated in many mitochondrial disorders, including LHON. The purpose of this study was to investigate the role of oxidative stress induced apoptosis in LHON.

Methods: The 2-deoxy-D-ribose induced apoptotic response of peripheral blood lymphocytes from six patients with LHON and six healthy subjects was investigated using light microscopy, flow cytometry, agarose gel electrophoresis, and the measurement of mitochondrial membrane potential.

Results: Cells of patients with LHON had a higher rate of apoptosis than those of controls and there was evidence of mitochondrial involvement in the activation of the apoptotic cascade.

Conclusions: These differences in oxidative stress induced apoptosis are in line with the hypothesis that redox homeostasis could play a role in the expression of genetic mutations in different individuals and could represent a potential target in the development of new therapeutic strategies.
\end{abstract}

L eber's hereditary optic neuropathy (LHON) is a late onset neurological disorder, in most cases associated with - specific mitochondrial DNA (mtDNA) point mutations. In some cases, a combination of two or more mutations has been reported. Eighteen different mutations have been described, three of which (nucleotides 11778, 3460, and 14484) are present in 95\% of families and have been considered pathogenetic (first class mutations). ${ }^{1}$ The clinical features of LHON include acute or subacute bilateral loss of central vision, which affects patients in the second or third decade of life. In addition to visual loss, patients and their maternal relatives have a variety of ancillary symptoms, such as cardiac conduction defects. ${ }^{2}$ Various minor neurological problems, including ataxia, sensory neuropathy, and brainstem evoked auditory response anomalies, have been reported in patients without other neurological findings. ${ }^{3}$ From the biochemical point of view, all patients with LHON show mitochondrial dysfunction in complex III or IV polypeptides, especially in complex $\mathrm{I}^{5}{ }^{6}$ associated with missense mutations in mtDNA. Experimental studies suggest that an inverse association exists between the activity of complex I and reactive oxygen species (ROS), ${ }^{78}$ agreeing with several lines of evidence indicating that ROS play a pivotal role in the pathogenesis of LHON. Therefore, a combination of complex I subunit mutations, partial deficiency of oxidative phosphorylation, as demonstrated by Guy et al, ${ }^{9}$ and increased production of ROS may be the pathological events culminating in cell death and optic neuropathy. However, many of the data on the pathogenesis of LHON suggest that mtDNA mutations may be necessary but not sufficient for the manifestation of the disease; an intriguing feature of LHON is that only $50 \%$ of men and $10 \%$ of women harbouring one of the three primary mutations develop optic neuropathy. ${ }^{10}$ This incomplete penetrance and the preference for male individuals suggest that nuclear or mitochondrial genes, or environmental factors, play a role in the pathogenesis of the disease, yet to be understood.
Recent studies have shown that the death of retinal ganglion cells (RGCs) characteristic of LHON occurs in an apoptotic manner, so that changes in the mitochondrial respiratory chain and/or in oxidative stress could play a role in the induction of apoptosis in LHON. ${ }^{112}$ Apoptosis or programmed cell death is a type of cell death different from necrosis, which is essential for the removal of excess, unwanted, and harmful cells and for the maintenance of homeostasis. ${ }^{13}$ Mitochondria play a key role in programmed cell death, namely: (1) they release cytochrome c into the cytosol in the first phase of apoptosis, activating caspase $9^{14}$; (2) bcl-2, a protein located in the outer mitochondrial membrane, belongs to a large family of proteins involved in apoptosis, and is thought to inhibit the process through the release of cytochrome $\mathrm{c}^{15}$; (3) the decrease of inner mitochondrial membrane potential, mediated by opening of the mitochondrial permeability transition pore, is a very early event in the apoptotic process ${ }^{16}$; (4) electron transport alterations, oxidative phosphorylation dysfunction, and storage of free radicals suggesting mitochondrial dysfunction have been shown in apoptotic cells. ${ }^{17}$

To investigate the role of oxidative stress in cells of patients with LHON, we studied oxidative stress induced apoptosis mediated by 2-deoxy-D-ribose (dRib) in peripheral blood lymphocytes.

\section{MATERIALS AND METHODS}

We analysed peripheral blood lymphocytes (PBLs) from six patients with LHON, comparing the results with those of six healthy age matched control. All patients had painless

Abbreviations: dRib, 2-deoxy-D-ribose; JC-1, 5, 5',6,6'-tetrachloro1,1',3,3'-tetraethylbenzimidazolcarbocyanine iodide; LHON, Leber's hereditary optic neuropathy; mt, mitochondrial; $\mathrm{ONH}$, optic nerve hypoplasia; PBL, peripheral blood lymphocyte; RGC, retinal ganglion cell; ROS, reactive oxygen species 
subacute subsequential visual loss (usually within eight weeks), with early fundoscopic peripapillary telangiectatic microangiopathy and subsequent optic atrophy. All patients underwent cardiological, neurophysiological, and neuroradiological examination, but no additional neurological or cardiac features were found. LHON was confirmed by the demonstration of an mtDNA mutation: five patients had the Gl1778A mutation and one had the T14484C mutation. Biochemical tests, including routine blood chemistry- notably concentrations of pyruvate, lactate, vitamin E, and vitamin A-were normal.

Peripheral blood lymphocytes from patients and controls were obtained in an aseptic manner; mononuclear cells were separated by centrifugation on a Lymphoprep gradient, ${ }^{18}$ and treated as previously reported. ${ }^{19}$ Apoptotic cell death was induced with dRib, a reducing sugar that stimulates apoptosis by oxidative stress. ${ }^{2021}$ dRib was added to a final concentration of $10 \mathrm{mM}$. The cells were incubated at $37^{\circ} \mathrm{C}$ for
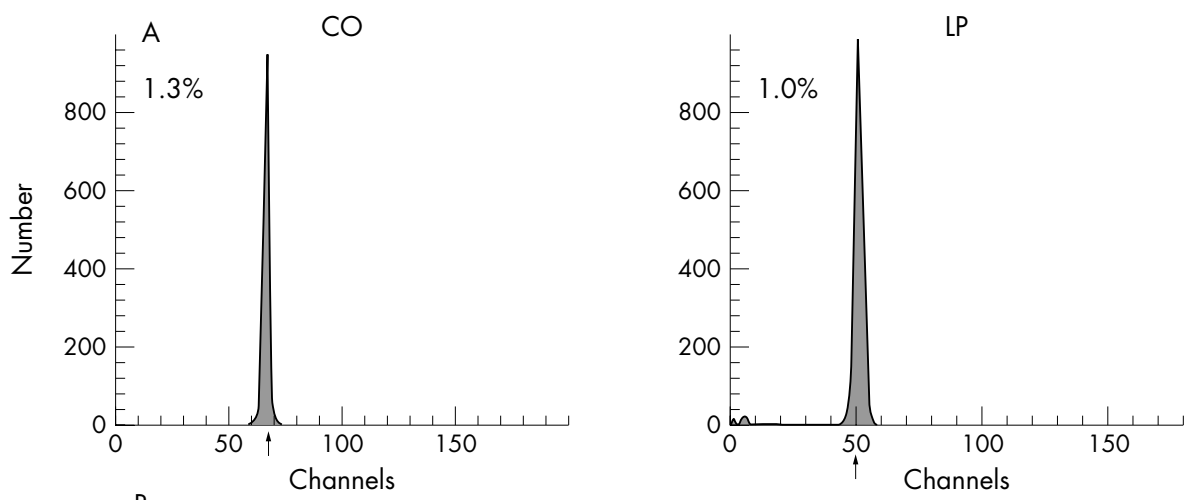

Figure 1 2-Deoxy-D-ribose (dRib) induced apoptosis in peripheral blood lymphocytes of patients and controls assessed by flow cytometric analysis of DNA content in the sub-G1 region. Cells were analysed after $(A)$ one, (B) 24, (C) 48 , and (D) 72 hours of incubation with DRib. CO, control; LP, patient with Leber's hereditary optic neuropathy (mutation G1 1778A). In each panel, the number refers to the percentage of apoptotic cells.
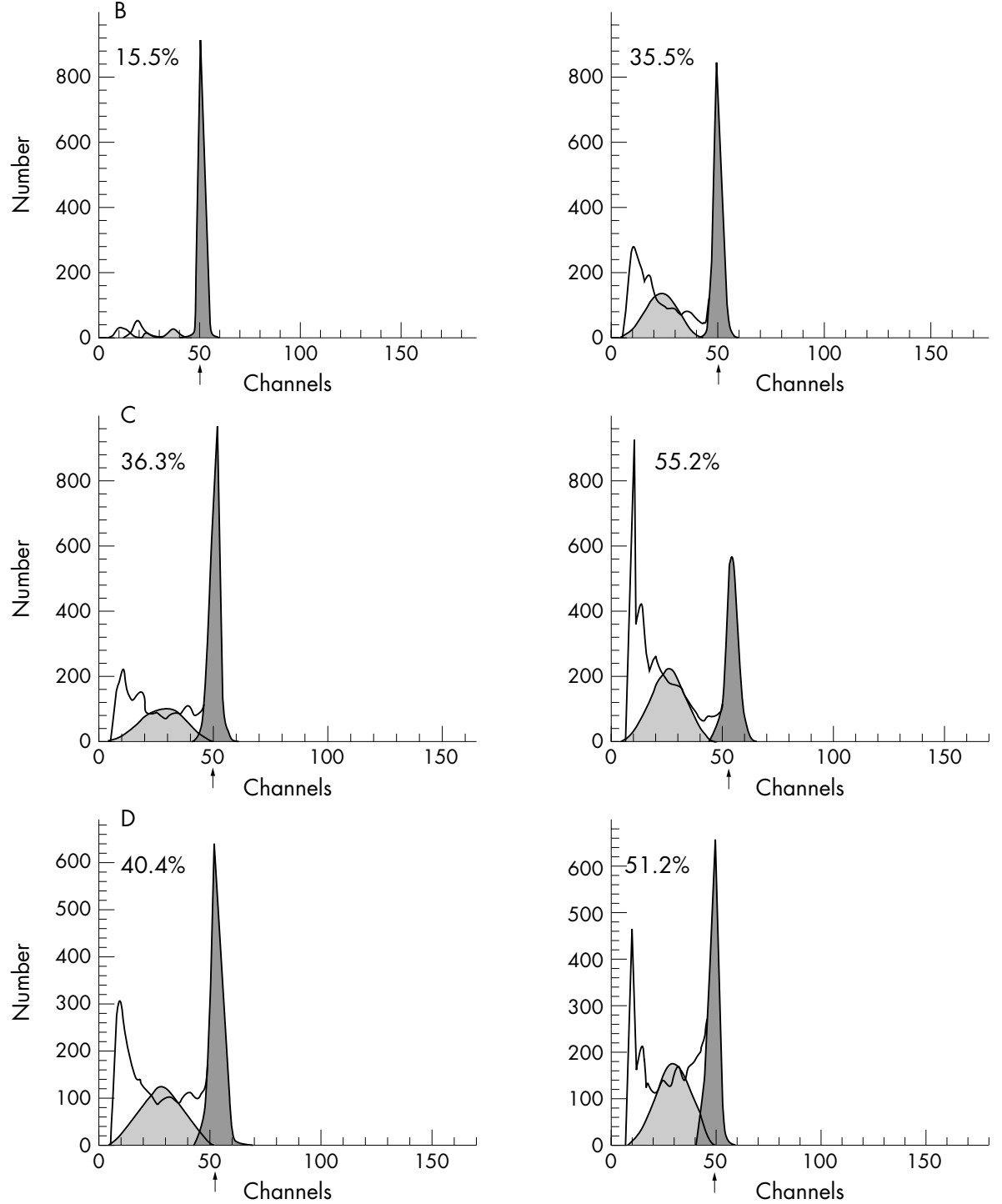
one, 24, 48, and 72 hours in a 5\% carbon dioxide atmosphere. At each time point, cells from each patient and control were harvested and analysed by light microscopy, flow cytometry, gel electrophoresis, and by measurement of mitochondrial membrane potential; morphological confirmation of apoptosis (cell shrinkage, nuclear condensation, extensive formation of membrane blebs, and apoptotic bodies) was observed by light microscope examination, as reported previously. ${ }^{22}$ The presence of apoptotic cells was evaluated by flow cytometry as reduced fluorescence of propidium iodide (a DNA binding dye) in the apoptotic nuclei, according to Nicoletti et al. ${ }^{23}$ Quantitative measurement of the time course and the extent of apoptosis in PBLs was performed primarily by the assessment of cells appearing in a sub-Gl peak on the DNA profiles. Reduced DNA binding of propidium iodide dye in apoptotic cells has been seen in several systems, ${ }^{24}$ including PBLs, and has been validated as a method for the quantitative analysis of the apoptotic response in PBLs. ${ }^{25}$

One of the major biochemical events of apoptosis is the internucleosomal cleavage of DNA strands ${ }^{26}$; this process results in DNA fragmentation into 200 base pairs, or multiples of them, and appears as a "ladder" pattern in agarose gel electrophoresis. Statistical analysis of the cytofluorimetric assay data was performed by the Kruskal Wallis test, a non-parametric test, taking p values less than 0.05 to be significant.

To delineate the mechanism of dRib cytotoxicity in PBLs, we analysed the dissipation of the mitochondrial membrane potential $\left(\Delta \Psi_{\mathrm{m}}\right)$ by a semiquantitative assay using a mitochondrion specific probe of the carbocyanine family: 5, 5' , 6, 6' - tetrachloro - 1, 1' , 3, 3' -tetraethylbenzimidazolcarbocyanine iodide (JC-1). ${ }^{27} \mathrm{JC}-1$ has been used successfully for flow cytometric measurement of mitochondrial potential by virtue of the fact that its dual emission characteristics are sensitive to membrane potential. ${ }^{28}$ It is mitochondrion selective, forming aggregates in normal polarised mitochondria that emit at $590 \mathrm{~nm}$ (red-orange) after excitation at $490 \mathrm{~nm}$. The monomeric form found in cells with depolarised mitochondrial membranes emits green fluorescence at $527 \mathrm{~nm}$. Determination of the JC-1 fluorescence ratio is a well established and reliable method to monitor changes in mitochondrial membrane potential. ${ }^{27}$ Cells incubated
A

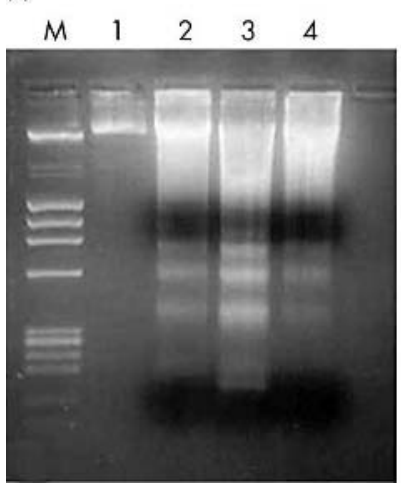

B

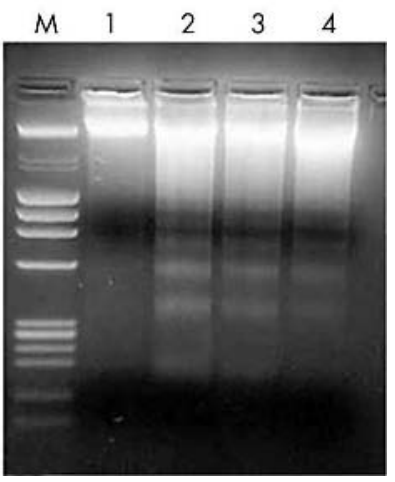

Figure 3 DNA gel electrophoresis of (A) Leber's hereditary optic neuropathy (mutation G1 1778A) and (B) control peripheral blood lymphocytes incubated with 2-deoxy-D-ribose. Lanes 1, one hour of incubation; lanes 2, 24 hours of incubation; lanes 3, 48 hours of incubation; lanes 4, 72 hours of incubation. $M$, molecular size marker

with and without dRib were stained with $10 \mu \mathrm{M}$ JC-1 for 30 minutes at room temperature and analysed by flow cytometry.

\section{RESULTS}

\section{Flow cytometry}

Quantitative analysis of the sub-Gl region of dRib treated cells showed a time dependent increase in the percentage of hypodiploid DNA in both groups. After one hour of incubation with dRib, the percentage of apoptotic cells was similar in the two groups, but after 24,48 , and 72 hours of culture, it was much higher in PBLs of patients with LHON than in those of normal donors $(\mathrm{p}<0.05$; fig 1$)$.

Between one and 72 hours of culture with dRib, the increase in percentage of apoptotic cells was greater in PBLs of patients with LHON (50.7 fold) than in those of controls ( 30.8 fold). The maximum difference in apoptotic response to dRib between the two cell populations was seen after 24 hours of incubation, when the percentage of apoptotic cells in the patients with LHON was more than double ( 2.2 fold) that of control cells. No significant difference was found between the two groups of PBLs cultured without dRib (fig 2).

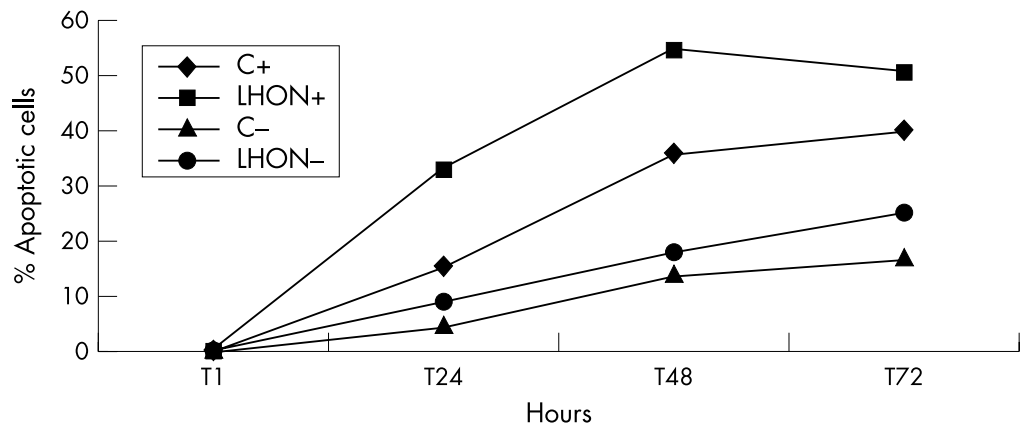

Figure 2 Cytofluorimetric analysis of apoptotic peripheral blood lymphocytes (PBLs) of six patients with Leber's hereditary optic neuropathy (LHON) and six controls cultured with $(+)$ and without (-) 2-deoxy-D-ribose. Cells were analysed after one, 24, 48, and 72 hours of culture. Values are expressed as mean (SD) percentage of apoptotic PBLs.

\begin{tabular}{|l|c|c|c|c|}
\hline & $\mathrm{T} 1$ & $\mathrm{~T} 24$ & $\mathrm{~T} 48$ & $\mathrm{~T} 2$ \\
\hline Controls (+) & $1.31(0.62)$ & $15.48(4.67)$ & $36.30(7.05)$ & $40.40(7.13)$ \\
\hline LHON patients (+) & $1.01(0.48)$ & $33.50(6.54)$ & $55.18(10.00)$ & $51.20(6.72)$ \\
\hline Controls (-) & $0.49(0.26)$ & $4.42(0.74)$ & $14.10(5.94)$ & $17.02(5.37)$ \\
\hline LHON patients (-) & $0.79(0.27)$ & $8.75(2.58)$ & $18.34(2.50)$ & $25.81(1.78)$ \\
\hline
\end{tabular}



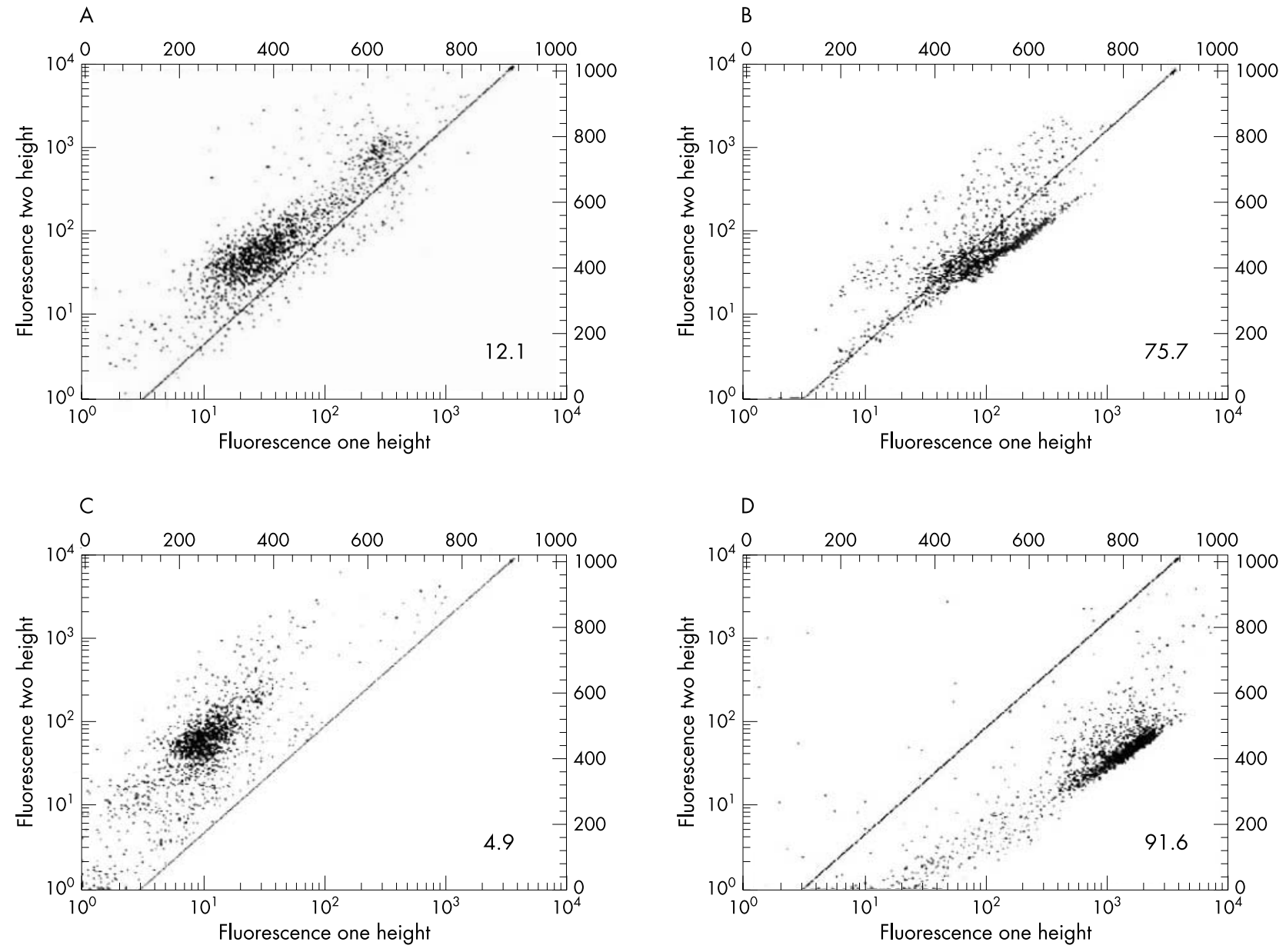

Figure 4 Changes in mitochondrial membrane potential in peripheral blood lymphocytes from (A, B) a control and (C, D) a patient with Leber's hereditary optic neuropathy (mutation G1 1778A) after one and 48 hours of incubation with 2-deoxy-D-ribose, as determined by uptake of JC-1. In the histograms of cells stained with JC-1, the labels "Fluorescence two height" for the ordinate and "Fluorescence one height" for the abscissa refer to the fluorescence readings of the two channels, FL2 $(590 \mathrm{~nm})$ and FL1 $(527 \mathrm{~nm})$, of the flow cytometer, respectively. Thus, cells with high red fluorescence (high $\Delta \psi \mathrm{m})$ are in the upper left hand of the panel of each histogram and those with low $\Delta \psi \mathrm{m}$ are in the lower right hand of the panel. The percentage at the lower right of each profile is the percentage of cells with low intensity fluorescence.

\section{Agarose gel electrophoresis}

The presence of apoptotic cells in dRib treated PBLs was confirmed using agarose gel electrophoresis, a semiquantitative method. However, in development situations in which apoptotic cells are scattered throughout a larger population of non-apoptotic cells, the demonstration of a DNA ladder may be difficult. ${ }^{29}$

Figure 3 shows DNA agarose gel electrophoresis of PBLs from patients with LHON (fig lA) and controls (fig 1B). Cells from patients with LHON incubated with dRib showed DNA fragmentation with a striking, typical "ladder pattern" after 24, 48, and 72 hours of culture. After 24 and 48 hours of incubation with dRib, control cells showed a weak "ladder configuration" (fig 1B). In both groups, agarose gel electrophoresis showed DNA fragmentation (smearing) after 24, 48, and 72 hours of culture without dRib, but not the typical ladder configuration (data not shown).

\section{Measurement of mitochondrial membrane potential}

The incubation of LHON and control cells with dRib induced a significant reduction in the JC-1 590/527 nm fluorescence ratio, which levelled off after 48 hours. Figure 4 shows a clear increase in the percentage of cells (lower right of panel) emitting green fluorescence after 48 hours of incubation with dRib; namely, cells with depolarised mitochondrial membranes. This increase was more evident in cells from patients with LHON (fig 4D) than in controls (fig 4B), with the percentage of cells emitting green fluorescence being $91.6 \%$ and $75.7 \%$, respectively.

\section{DISCUSSION}

LHON is a maternally inherited form of central vision loss, in which three prevalent pathogenic mtDNA mutations at positions 11778,3460 , and 14484 affecting different subunits of complex I cause RGC death and optic nerve atrophy. Cell death is painless and without inflammation, suggesting an apoptotic mechanism. Recently, the role of apoptosis in RGC degeneration has been tested extensively; Krishanamoorthy et al showed that in an immortalised rat RGC cell line, deprivation of trophic factors induced cellular death by apoptosis. ${ }^{30}$ Wein and Levin ${ }^{31}$ found that transection axotomy) of the optic nerve in small animals induces retrograde axonal degeneration and cell death by apoptosis of RGCs. Activation of the apoptotic cascade in retinal neurones appears to occur via the major apoptotic pathway described for neurones of the central nervous system, including activation of caspases, (mainly caspases 9 and 3), ${ }^{32}$ c-jun kinase, $^{33}$ and $\mathrm{Bcl}$ family proteins. ${ }^{34}$ In addition to these proteins, other molecules, such as tumour necrosis factor $\alpha^{35}$ and glutamate, ${ }^{36}$ have been shown to induce apoptosis in retinal neurones. Several authors have evaluated different aspects of apoptosis in tissue and cells from patients with 
LHON. Saadati et al compared the distinctive patterns of nerve fibre distribution and axonal dropout in LHON and other inherited disorders, such as optic nerve hypoplasia $(\mathrm{ONH})$, and concluded that $\mathrm{ONH}$ is the result of an apoptotic process, whereas LHON is the result of a specific degenerative process. ${ }^{37}$ However, this postmortem study had two important limitations: only one nerve was observed and this was done 60 years after the onset of LHON. Mirabella and colleagues $^{38}$ evaluated apoptosis in muscle biopsies of patients with different forms of mitochondrial encephaloneuromyopathies, and reported abnormalities in the process in all cases except a LHON specimen characterised by the absence of a detectable biochemical or morphological abnormality; however, only one case of LHON disease was examined in that study. Various results have recently been obtained; Danielson and colleagues ${ }^{39}$ were the first to discover that cells (osteosarcoma derived cybrid) with pathogenic LHON mutations were more sensitive to Fas dependent apoptosis than were control cells. Ghelli et al recently stressed the role of apoptosis in the same cell lines harbouring one of the three most frequent LHON pathogenic mutations. ${ }^{12}$ They documented that LHON cybrid cell death is apoptotic and saw increased release of cytochrome c into the cytosol, demonstrating mitochondrial involvement in the activation of the apoptotic cascade.

Other studies have provided direct proof that oxidative stress can damage mtDNA. In two recent papers, oxidative stress was engineered genetically in mice by targeted deletions in superoxide dismutase or the adenine nuclear transporter. Subsequent analysis showed a significant increase in mitochondrial rearrangements, associated with impaired mitochondrial function and morphology. ${ }^{40}{ }^{41}$ Wong et al found that mtDNA mutations such as LHON mutations confer sensitivity to oxidative stress induced death. ${ }^{42}$ In a cell model with complex I impairment, Barrientos and Moraes ${ }^{7}$ identified a positive and quantitative correlation between apoptosis and free radical production. In 2002, Wong and colleagues $^{11}$ and Ghelli et al, ${ }^{12}$ in two different experiments, showed that cybrids with LHON mutation have increased production of ROS. This suggested the reason for the cell specificity of the LHON degeneration phenotype for the first time, highlighting that degeneration of RGCs could be the result of an interaction between mtDNA mutation, complex I alteration, and increased ROS production. In an elegant experiment with mice in 2003, Qi and colleagues $^{43}$ induced the reduction of mitochondrial superoxide dismutase and observed similar histopathological findings to those seen in the RGC of patients with LHON, confirming the key role of ROS in the pathogenesis of LHON.

Our study is the first one in which apoptosis induced by oxidative stress has been examined in cells from patients with LHON. Lymphocytes from patients with LHON treated with the oxidising agent dRib showed a significant increase in the percentage of apoptotic cells with respect to controls; no relation was evident between the percentage of apoptotic cells and the type of mtDNA mutation. The JC-1 test revealed depolarisation of the mitochondrial membrane potential in lymphocytes from patients with LHON, with a greater shift from red-orange to green fluorescence after 48 hours of culture than in control cells. Our observation confirms that the apoptotic process induced by oxidative stress primarily involves the mitochondrial cascade. LHON cells showed a particular susceptibility to this inducer and confirmed the notion of a direct link between complex I (commonly altered in patients with LHON) and changes in mitochondrial membrane permeability. Fontaine et al found that complex I may be part of the pore complex, strongly supporting this association. $^{44}$ Finally, our data are in line with and complementary to those from LHON cybrid cells, ${ }^{11}{ }^{12}$ confirming that the alteration of redox homeostasis renders the RGCs of patients with LHON vulnerable to apoptotic cell death. This factor could play a role in the different individual expression of genetic mutation and be a potential target in the development of new therapeutic strategies.

\section{ACKNOWLEDGEMENTS}

This research was partly financed by a grant from Siena University to $\mathrm{CB}$ and from the Ministry of Health to AF.

\section{Authors' affiliations}

C Battisti, P Formichi, E Cardaioli, S Bianchi, A Federico, Department of Neurological and Behavioural Sciences, University of Siena, 53100 Siena, Italy

P Mangiavacchi, S A Tripodi, P Tosi, Department of Pathology, University of Siena

Competing interests: none declared

\section{REFERENCES}

1 Riordan-Eva P, Harding AE. Leber's hereditary optic neuropathy: the clinical relevance of different mitochondrial DNA mutations. J Med Genet 1995:32:81-7.

2 Nikoskeleainen E, Wanne O, Dahl M. Pre-excitation syndrome and Leber's hereditary optic neuroretinopathy [letter]. Lancet 1985;23:696.

3 Paulus W, Straube A, Bauer W, et al. Central nervous system involvement in Leber's optic neuropathy. J Neurol 1991;240:251-3.

4 Mondelli M, Rossi A, Scarpini C, et al. Leber's optic atrophy: VEP and BAEP changes in 16 asymptomatic subjects. Acta Neurol Scand 1991;84:366.

5 Brown MD, Voljavec AS, Lott MT, et al. Mitochondrial DNA complex I and III mutations associated with Leber's hereditary optic neuropathy. Genetics 1992;130:163-73.

6 Johns DR, Neufeld MJ. Cytochrome c oxidase mutations in Leber hereditary optic neuropathy. Biochem Biophys Res Commun 1993;196:810-15.

7 Barrientos A, Moraes CT. Titrating the effect of mitochondrial complex 1 impairment in the cell physiology. J Biol Chem 1999;274:16188-97.

8 Cortopassi G, Wong E. Modelling the effects of age-related mtDNA mutation accumulation; complex I deficiency, superoxide and cell death. Biochim Biophys Acta 1999;1271:171-6.

9 Guy J, Qi X, Pallotti F, et al. Rescue of a mitochondrial deficiency causing Leber hereditary optic neuropathy. Ann Neurol 2002;52:534-42.

10 Man PYW, Turnbull DM, Chinnery PF. Leber hereditary optic neuropathy. J Med Genet 2002;39:162-9.

11 Wong A, Cavelier L, Collins-Schramm HE, et al. Differentiation-specific effects of LHON mutations introduced into neuronal NT2 cells. Hum Mol Genet 2002;11:431-8.

12 Ghelli A, Zanna C, Porcelli AM, et al. Leber's hereditary optic neuropathy (LHON) pathogenic mutations induce mitochondrial-dependent apoptosis death in transmitochondrial cells incubated with galactose medium. J Biol Chem 2003;278:4145-50. Epub 2002 Nov.

13 Granville DJ, Carthy CM, Hunt DV, et al. Apoptosis-molecular aspects of cell death and disease. Lab Invest 1998;78:893-913.

14 Bossy-Wetzel E, Green DR. Apoptosis: checkpoint at the mitochondrial frontier. Mutat Res 1999;434:243-51

15 Adachi S, Cross AR, Babior BM, et al. Bcl-2 and the outer mitochondrial membrane in the inactivation of cytochrome $\mathrm{c}$ during Fas-mediated apoptosis. J Biol Chem 1997;29:21878-2.

16 Susin SA, Zamzami N, Castedo M, et al. Bcl-2 inhibits the mitochondrial release of an apoptogenic protease. J Exp Med 1996;184:1331-41.

17 Greenlund LJS, Korsmeyer SJ, Johnson EM Jr. Role of Bcl-2 in the survival and function of developing and mature sympathetic neurons. Neuron 1995; 14:303-15.

18 Boyum A. Separation of lymphocytes, granulocytes and monocytes from human blood using iodinated density gradient media. Methods Enzymol 1984; 108:88-102.

19 Battisti C, Formichi P, Tripodi SA, et al. Increased apoptotic response to 2-deoxy-D-ribose in ataxia-telangiectasia. J Neurol Sci 1996;144:128-34.

20 Formichi P, Battisti C, Tripodi SA, et al. Apoptotic response and cell cycle transition in ataxia-telangiectasia cells exposed to oxidative stress. Life Sci 2000;66: 1893-903.

21 Battisti C, Formichi P, Tripodi SA, et al. Enhanced 2-deoxy-D-ribose-inducedapoptosis, a phenotype of lymphocytes from old donors, not observed in the Werner syndrome. Exp Gerontol 2000;35:605-12.

22 Kerr JFR, Harmon BV. Definition and incidence of apoptosis: an historical perspective. In: Tomei LD, Cope FO, eds. Apoptosis: the molecular basis of cell death. New York: Cold Spring Harbor Laboratory Press, Plainview, 1991:5-29.

23 Nicoletti I, Migliorati G, Pagliacci MG, et al. A rapid and simple method for measuring thymocyte apoptosis by propidium iodide staining and flow cytometry. J Immunol Methods 1991;139:271-9.

24 Telford WG, King LE, Fraker PJ. Evaluation of glucocorticoid induced DNA fragmentation in mouse thymocytes by flow cytometry. Cell Prolif $1991 ; 24: 447-59$ 
25 Barbieri D, Troiano L, Grassilli E, et al. Inhibition of apoptosis by zinc: a reappraisal. Biochem Biophys Res Commun 1992; 187:1256-61

26 Arends MJ, Wyllie AH. Apoptosis: mechanisms and roles in pathology. Int Rev Exp Pathol 1991;32:223-54.

27 Cossarizza A, Baccarani Contrl M, Kalashnikova G, et al. A new method for the cytofluorimetric analysis of mitochondrial membrane potential using the J-aggregate forming lipophilic cation $5,5^{\prime}, 6,6^{\prime}$-tetrachloro-1, $1^{\prime}, 3,3^{\prime}$ tetraethylbenzimidazolcarbocyanine iodide (JC-1). Biochem Biophys Res Commun 1993;197:40-5.

28 Chiu SM, Oleinick NL. Dissociation of mitochondrial depolarization from cytochrome $c$ release during apoptosis induced by photodynamic therapy. Br J Cancer 2001;84:1098-108.

29 Sanders EJ. Methods for detecting apoptotic cells in tissues. Histol Histopathol 1997;12:1169-77.

30 Krishnamoorthy RR, Agarwal P, Prasanna G, et al. Characterization of a transformed rat retinal ganglion cell line. Brain Res Mol Brain Res 2001;86:1-12

31 Wein FB, Levin LA Current understanding of neuroprotection in glaucoma. Curr Opin Ophthalmol 2002;13:61-7.

32 Kermer $\mathbf{P}$, Ankerhold R, Klocher N, et al. Caspase-9: involvement in secondary death of axotomized rat retinal ganglion cells in vivo. Brain Res Mol Brain Res, 2000;85: 144-50.

33 Grimm C Wenzel A, Hafezi $F$ et al Gene expression in the mouse retina: the effect of damaging light. Mol Vis 2000;6:252-60.

34 Chaum E. Retinal neuroprotection by growth factors: a mechanistic perspective. J Cell Biochem 2003;88:57-75.
35 Tezel G, Li LY, Patil RV, et al. TNF-alpha and TNF-alpha receptor-1 in the retina of normal and glaucomatous eyes. Invest Ophthalmol Vis Sci 2001;42:1787-94

36 Izumi Y, Shimamoto K, Benz AM, et al. Glutamate transporters and retinal excitotoxicity. Glia 2002;39:58-68.

37 Saadati HG, Hsu HY, Heller HB, et al. A histopathologic and morphometric differentiation of nerves in optic nerve hypoplasia and Leber hereditary optic neuropathy. Arch Ophthalmol 1998;116:911-16.

38 Mirabella M, Di Giovanni S, Silvestri G, et al. Apoptosis in mitochondrial encephalomyopathies with mitochondrial DNA mutations: a potential pathogenic mechanism. Brain 2000;123:93-104.

39 Danielson SR, Wong A, Carelli V, et al. Cells bearing mutations causing Leber's hereditary optic neuropathy are sensitive to Fas-induced apoptosis. $J$ Biol Chem 2002;277:5810-15.

40 Esposito LA, Melov S, Panov A, et al. Mitochondrial disease in mouse results in increased oxidative stress. Proc Natl Acad Sci U S A 1999;96:4820-5.

41 Melov S, Coskun P, Patel M, et al. Mitochondrial disease in superoxide dismutase 2 mutant mice. Proc Natl Acad Sci U S A 1999;96:846-51.

42 Wong A Cortopassi G. mtDNA mutations confer cellular sensitivity to oxidant stress that is partially rescued by calcium depletion and cyclosporin $A$. Biochem Biophys Res Commun 1997;239:139-45.

43 Qi X, Lewin AS, Hauswirth WW, et al. Optic neuropathy induced by reduction in mitochondrial superoxide dismutase. Invest Ophthalmol Vis Sci 2003:44:1088-96.

44 Fontaine $E$, Eriksson $O$, Ichas $F$, et al. Regulation of the permeability transition pore in skeletal muscle mitochondria. Modulation by electron flow through the respiratory chain complex 1. J Biol Chem 1998;273:12662-8. 